\title{
BMJ Open Urinary albumin-to-creatinine ratio levels are associated with subclinical atherosclerosis and predict CVD events and all-cause deaths: $a$ prospective analysis
}

To cite: Liu S, Niu J, Wu S, et al. Urinary albumin-tocreatinine ratio levels are associated with subclinical atherosclerosis and predict CVD events and all-cause deaths: a prospective analysis. BMJ Open 2021;11:e040890. doi:10.1136/ bmjopen-2020-040890

- Prepublication history for this paper is available online. To view these files, please visit the journal online (http://dx.doi. org/10.1136/bmjopen-2020040890).

SL, JN and SW contributed equally.

Received 25 May 2020 Revised 29 December 2020 Accepted 22 January 2021

Check for updates

(C) Author(s) (or their employer(s)) 2021. Re-use permitted under CC BY-NC. No commercial re-use. See rights and permissions. Published by BMJ.

For numbered affiliations see end of article.

Correspondence to

Dr Mian Li;

limian39@aliyun.com,

DrYuXu;

jane.yuxu@gmail.com and

Dr Yufang Bi;

byf10784@rjh.com.cn

\section{ABSTRACT}

Objective We aimed to examine the associations of urinary albumin-to-creatinine ratio (ACR) levels with risks of subclinical atherosclerosis, cardiovascular events and all-cause deaths.

Methods Data from a large population-based cohort were used, which included 9580 participants aged $\geq 40$ years free from cardiovascular diseases. Carotid intimamedia thickness, brachial-ankle pulse wave velocity and ankle-brachial index were measured at baseline to assess subclinical atherosclerosis. After a median of 4.53 years' follow-up, 486 cardiovascular events and 230 all-cause deaths were recorded.

Results The urinary ACR levels were categorised into three groups. Compared with the normal group ( $0 \leq A C R$ $<7.82 \mathrm{mg} / \mathrm{g}$ ), people with low-grade albuminuria $(7.82 \leq \mathrm{ACR}<30 \mathrm{mg} / \mathrm{g})$ and albuminuria $(\mathrm{ACR} \geq 30 \mathrm{mg} / \mathrm{g})$ had higher levels of subclinical atherosclerosis. In prospective analysis, people with low-grade albuminuria was not significantly associated with cardiovascular events ( $\mathrm{HR}=1.18$; $95 \% \mathrm{Cl} 0.95$ to 1.46], whereas people with albuminuria had a $50 \%$ higher risk of cardiovascular events ( $\mathrm{HR}=1.50 ; 95 \% \mathrm{Cl} 1.11$ to 2.03). People with lowgrade albuminuria and albuminuria had $43 \%(\mathrm{HR}=1.43$; $95 \% \mathrm{Cl} 1.05$ to 1.93$)$ and $87 \%(\mathrm{HR}=1.87 ; 95 \% \mathrm{Cl} 1.24$ to 2.81) higher risks of all-cause deaths during follow-up, respectively. In stratified analysis, the association of higher ACR with risks of cardiovascular events and all-cause deaths was stronger among individuals with concomitant subclinical atherosclerosis, the presence of diabetes and more cardiovascular risk factors, respectively. Conclusions ACR levels were positively associated with subclinical atherosclerosis and predicted the risks of cardiovascular events and all-cause deaths. Evaluation of ACR levels should be integrated into risk stratification and prevention of cardiovascular events and all-cause deaths, especially among those with preexisting subclinical atherosclerosis and cardiometabolic abnormalities.
Strengths and limitations of this study

- It provided a comprehensive evaluation of microvascular and macrovascular indicators and detected the joint impact on the risks of cardiovascular events and all-cause deaths in general population.

- Cross-sectional analysis was applied to investigate the associations between albumin-to-creatinine ratio (ACR) levels and subclinical atherosclerosis, which could not show direct causal links.

- The follow-up duration was comparatively short and only one follow-up visit was conducted.

- A relatively small number of participants with cardiovascular events and all-cause deaths were included, so composite outcomes were adopted.

- Urinary ACR levels were calculated using a single morning urine sample, instead of 24-hour urine sample.

\section{INTRODUCTION}

Albumin-to-creatinine ratio (ACR) well represents the severity of proteinuria and is a marker of endothelial dysfunction. Albuminuria were closely associated with the risks of cardiovascular diseases (CVD). ${ }^{1}{ }^{2}$ Further explorations found that higher level of ACR within the normal range was also associated with an increased risk of cardiovascular events. ${ }^{3}$

In China, the prevalence of CVD is still on the rise. ${ }^{4}$ Subclinical atherosclerosis, including but not limited to elevated carotid intima-media thickness (CIMT), carotid plaques, elevated brachial-ankle pulse wave velocity (Ba-PWV), abnormal ankle-brachial index (ABI), can reflect an elevated risk of atherosclerotic cardiovascular abnormalities and lead to major adverse CVD 


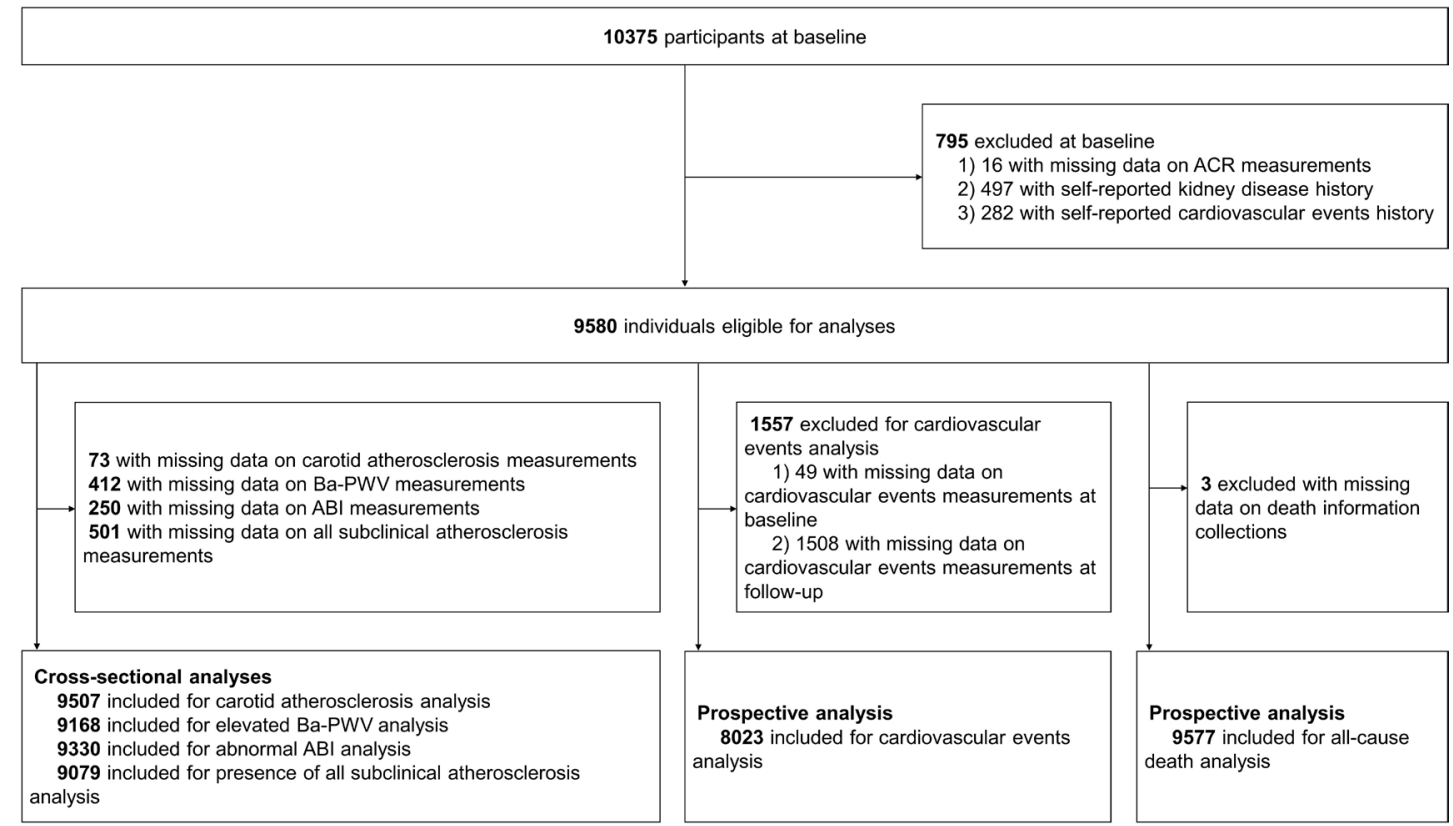

Figure 1 Study flowchart. Subclinical atherosclerosis: (1) carotid atherosclerosis (carotid intima-media thickness $>0.6 \mathrm{~mm}$ or the presence of carotid stenosis as $50 \%)$, (2) elevated Ba-PWV (Ba-PWV $>1789 \mathrm{~cm} / \mathrm{s}$ ) or (3) abnormal $\mathrm{ABI}(\mathrm{ABI}<0.9$ or $\mathrm{ABI}>1$.4). Cardiovascular events: the first instance of nonfatal myocardial infarction, nonfatal stroke, congestive heart failure or cardiovascular-related deaths. ABI, ankle-brachial index; $\mathrm{ACR}$, albumin-to-creatinine ratio; Ba-PWV, brachial-anklepulse wave velocity.

such as myocardial infarction, stroke, heart failure and cardiovascular-related deaths. Thus, early identification and prevention of subclinical atherosclerosis are of great importance.

Several previous studies have reported that a higher level of albuminuria was a risk factor for the development of atherosclerosis. ${ }^{5}$ There is also emerging evidence that low-grade albuminuria was significantly associated with subclinical atherosclerosis. ${ }^{6-8}$ However, studies that investigated the effect of ACR level on the risks of cardiovascular events and all-cause deaths by different status of subclinical atherosclerosis are still limited.

The main objective of our study was to comprehensively examine the cross-sectional associations of ACR levels with subclinical atherosclerosis indicators and further to detect the effect of ACR levels on the risks of cardiovascular events and all-cause deaths by subclinical atherosclerosis status, diabetes status and the number of cardiovascular risk factors during a median of 4.53 years' follow-up in a prospective cohort with general population in Shanghai, China.

\section{METHODS}

\section{Study population}

This study enrolled participants aged 40 years or older in Jiading District, Shanghai, China. The study design had been published elsewhere. ${ }^{9}$ At baseline (MarchAugust, 2010), 10375 registered permanent residents participated. At the follow-up visit (August 2014 to May 2015), 10372 responded. The inclusion and exclusion criteria are shown in figure 1 . Overall, we excluded
795 people with missing data on ACR measurements, self-reported kidney disease history or self-reported cardiovascular event history. After that, 9580 individuals were included. Furthermore, we excluded people with missing data and leave 9507 (carotid atherosclerosis), 9168 (Ba-PWV) and 9330 (ABI) participants for the analysis of individual subclinical atherosclerosis indicator, respectively. For prospective analysis, subjects with missing data at follow-up were further excluded, leaving 8023 participants for the analysis of cardiovascular events and 9577 for all-cause deaths.

The study protocols were approved by the Committee on Human Research at Rui-jin Hospital affiliated to Shanghai Jiaotong University School of Medicine. Each subject provided written informed consent.

\section{Baseline data collection and definition}

Urine albumin was measured with an immunoturbidimetric method (Beijing Atom High-Tech, Beijing, China) and was indexed to urine creatinine (Jaffe's kinetic method, Hitachi 7600-020, Tokyo, Japan) to estimate the urinary ACR. ACR levels $(\mathrm{mg} / \mathrm{g})$ were categorised into three groups: normal $(0 \leq \mathrm{ACR}<7.82)$, lowgrade albuminuria $(7.82 \leq \mathrm{ACR}<30)$ and albuminuria (ACR $\geq 30)$. The value 7.82 is the 75 th percentile value within the normal range of ACR $(0-30) .{ }^{10}$ Metabolic equivalent minutes per week (MET-min/week) were calculated and physical activity was dichotomised as meeting ( $\geq 600$ MET-min/week) or not meeting current guidelines. $^{11}$

Diabetes mellitus was defined as a fasting blood glucose $\geq 126 \mathrm{mg} / \mathrm{dL}$ and/or a postload glucose 


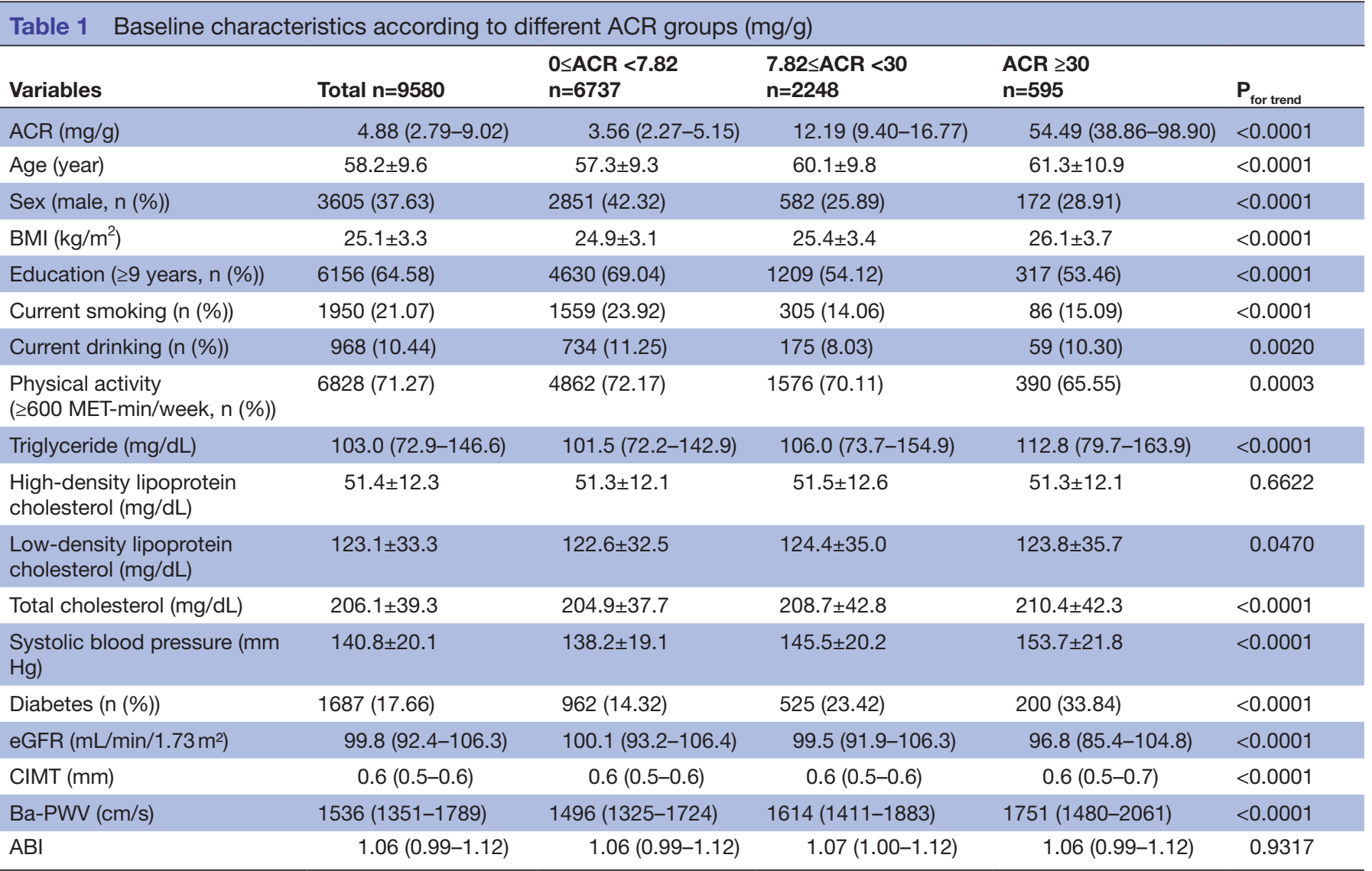

Continuous variables were presented as means \pm standard deviation or medians (quartile ranges); categorised variables were presented as $\mathrm{n}$ (\%). $\mathrm{ABI}$, ankle-brachial index; ACR, albumin-to-creatinine ratio; Ba-PWV, brachial-ankle pulse wave velocity; BMI, body mass index; CIMT, carotid intimamedia thickness; eGFR, estimated glomerular filtration rate; MET-min/week, metabolic equivalent minutes per week.

$\geq 200 \mathrm{mg} / \mathrm{dL}$ and/or haemoglobin A1c $\geq 6.5 \%$ and/or taking glucose-lowering medications. Six cardiovascular health (CVH) metrics including smoking habit, physical activity, body mass index (BMI), blood pressure, total cholesterol and fasting blood glucose were used for the assessment of ideal CVH. Each metric was categorised into ideal and nonideal levels according to the American Heart Association (AHA) definition. ${ }^{12}$ People with a nonideal level of each CVH was defined as having one cardiovascular risk factor.

Trained sonographer operated a high-resolution B-mode tomographic ultrasound system (Esaote Biomedica SpA, Italy) with a linear 7.5 MHz transducer to measure CIMT and to detect carotid plaques. Online CIMT value was captured and the larger value of both two sides was adopted for analysis. Multiaspect plaque detections were performed at sections of carotid arteries. CIMT $>0.6 \mathrm{~mm}$ (75th percentile value) or the presence of carotid stenosis as $50 \%$ was defined as carotid atherosclerosis.

Ba-PWV and ABI were determined with Colin VP-1000 (Model BP203RPE II, form PWV/ABI) by practitioners. The larger Ba-PWV value and two ABI values of both the left and right sides were adopted for analysis. Elevated Ba-PWV was defined as greater that the 75 th percentile value $(1789 \mathrm{~cm} / \mathrm{s})$ of the study population. Abnormal $\mathrm{ABI}$ was defined as $\mathrm{ABI}<0.9$ or $\mathrm{ABI}>1.4$.

A composite status of subclinical atherosclerosis was evaluated based on the presence of abnormal indicators and defined as subclinical atherosclerosis when any of the following criteria were met: (1) carotid atherosclerosis (CIMT $>0.6 \mathrm{~mm}$ or the presence of carotid stenosis as 50\%), (2) elevated Ba-PWV (Ba-PWV $>1789 \mathrm{~cm} / \mathrm{s}$ ), (3) abnormal $\mathrm{ABI}(\mathrm{ABI}<0.9$ or $\mathrm{ABI}>1.4)$.

\section{Outcome collection and definition}

Information on cardiovascular events, death and its specific cause was collected from local disease and death registries of the National Disease Surveillance Point System and National Health Insurance System. Over the study period, subjects' medical records would be collected and adjudicated centrally if they visited an emergency room or were hospitalised.

Cardiovascular events were defined as the first instance of nonfatal myocardial infarction, nonfatal stroke, congestive heart failure or deaths with established unequivocal cardiovascular cause during follow-up. All-cause deaths were defined as demise irrespective of any cause during follow-up. 
Table 2 Proportions of subclinical atherosclerosis, incidences of cardiovascular events and all-cause deaths according to different ACR groups (mg/g)

\begin{tabular}{|c|c|c|c|c|c|}
\hline $\begin{array}{l}\text { Proportions and } \\
\text { incidences (\%) }\end{array}$ & Total & $0 \leq \mathrm{ACR}<7.82$ & $7.82 \leq \mathrm{ACR}<30$ & $A C R \geq 30$ & $\mathbf{P}_{\text {for trend }}$ \\
\hline \multicolumn{6}{|c|}{ Proportions of subclinical atherosclerosis at baseline } \\
\hline $\begin{array}{l}\text { Carotid } \\
\text { atherosclerosis }\end{array}$ & $27.22 \%(2588 / 9507)$ & $24.94 \%(1670 / 6697)$ & $32.03 \%(712 / 2223)$ & $35.09 \%(206 / 587)$ & $<0.0001$ \\
\hline Elevated Ba-PWV & $24.98 \%(2290 / 9168)$ & $20.63 \%(1331 / 6453)$ & $32.51 \%(698 / 2147)$ & $45.95 \%(261 / 568)$ & $<0.0001$ \\
\hline Abnormal ABI & $6.82 \%(636 / 9330)$ & $6.66 \%(437 / 6566)$ & $6.63 \%(145 / 2186)$ & $9.34 \%(54 / 578)$ & 0.0820 \\
\hline \multicolumn{6}{|c|}{ Incidences of cardiovascular events and all-cause deaths during follow-up } \\
\hline Cardiovascular events & $6.06 \%(486 / 8023)$ & $5.00 \%(281 / 5620)$ & $7.74 \%(146 / 1886)$ & $11.41 \%(59 / 517)$ & $<0.0001$ \\
\hline All-cause deaths & $2.40 \%(230 / 9577)$ & $1.75 \%(118 / 6735)$ & $3.43 \%(77 / 2247)$ & $5.88 \%(35 / 595)$ & $<0.0001$ \\
\hline
\end{tabular}

Subclinical atherosclerosis: (1) carotid atherosclerosis (carotid intima-media thickness $>0.6 \mathrm{~mm}$ or the presence of carotid stenosis as $50 \%$ ), (2) elevated Ba-PWV (Ba-PWV $>1789 \mathrm{~cm} / \mathrm{s})$ or (3) abnormal $\mathrm{ABI}(\mathrm{ABI}<0.9$ or $\mathrm{ABI}>1.4)$. Cardiovascular events: the first instance of nonfatal myocardial infarction, nonfatal stroke, congestive heart failure or cardiovascular-related deaths.

$\mathrm{ABI}$, ankle-brachial index; ACR, albumin-to-creatinine ratio; Ba-PWV, brachial-ankle pulse wave velocity.

\section{Statistical analysis}

Continuous variables in normal distribution were presented as means \pm SD. Skewed variables were presented as medians (quartile ranges) and logarithmically transformed before analysis. $\chi^{2}$ tests were used to assess the proportions of subclinical atherosclerosis and incidences of cardiovascular events and all-cause deaths according to different ACR groups. Logistic regression models were adopted to examine the cross-sectional associations between ACR levels and subclinical atherosclerosis. Multivariate calibration models adjusted age, sex, BMI, education level, current smoking, current drinking, physical activity, triglyceride, high-density lipoprotein cholesterol, low-density lipoprotein cholesterol, total cholesterol, systolic blood pressure, diabetes status and estimated glomerular filtration rate (eGFR). Cox proportional hazards regression models were adopted to prospectively examine the associations between ACR levels and risks of cardiovascular events and all-cause deaths. In addition, we conducted stratified analysis to evaluate the effect of ACR levels on the risks of cardiovascular events and all-cause deaths by subclinical atherosclerosis status, diabetes status and number of cardiovascular risk factors.

A two-sided $p$ value $<0.05$ was considered statistically significant. All analyses were performed with SAS software V.9.4 (SAS Institute, Cary, North Carolina, USA).

\section{Patient and public involvement}

This research was done without patient involvement. Patients were not invited to comment on the study design or contribute to the acquisition, analysis or interpretation of data for the work. Patients were not consulted to develop relevant outcomes. Patients were not invited to draft the manuscript for integrity or accuracy.

\section{RESULTS}

Table 1 summarised baseline characteristics of this population. Among 9580 participants (men, 3605, 37.63\%), there were 6737,2248 and 595 subjects according to different ACR groups. With an increase in ACR level, age, BMI, triglyceride, low-density lipoprotein cholesterol, total cholesterol, systolic blood pressure, CIMT and Ba-PWV levels increased, while the eGFR level decreased, gradually $\left(\mathrm{p}_{\text {for trend }}<0.05\right)$. Proportions of men, high level of education, current smoking, current drinking and physical activity $(\geq 600$ MET-min/week) showed decreasing trends, except diabetes $\left(\mathrm{p}_{\text {for trend }}<0.05\right)$. Highdensity lipoprotein cholesterol and ABI showed no significant differences through ACR groups.

Proportions of subclinical atherosclerosis and incidences of cardiovascular events and all-cause deaths according to different ACR groups are presented in table 2. Along with the ACR level increasing, proportions of carotid atherosclerosis, elevated Ba-PWV and presence of subclinical atherosclerosis statistically increased and showed rising trends $\left(\mathrm{p}_{\text {for trend }}<0.05\right.$ ), while the proportions of abnormal ABI showed faint differences $\left(\mathrm{p}_{\text {for trend }}=0.08\right)$. Incidences of cardiovascular events were $5.00 \%, 7.74 \%$ and $11.41 \%$ in three groups, respectively. All-cause deaths incidences were $1.75 \%, 3.43 \%$ and $5.88 \%$, respectively. Significant differences were also observed and showed rising trends $\left(\mathrm{p}_{\text {for trend }}<0.05\right)$ across three groups.

Positive associations were found between ACR levels and subclinical atherosclerosis at baseline (table 3). Compared with the normal group, ORs (95\% CI) were 1.22 (1.07 to 1.38) for carotid atherosclerosis and 1.15 (1.02-1.30) for the presence of subclinical atherosclerosis among subjects with low-grade albuminuria after fully adjustments, respectively. Similar results were found for elevated Ba-PWV (OR=1.44; 95\% CI 1.27 to 1.64$)$ after 
Table 3 Associations between ACR levels and subclinical atherosclerosis at baseline (with reference to $0 \leq \mathrm{ACR}<7.82, \mathrm{mg} / \mathrm{g}$ )

\begin{tabular}{|c|c|c|c|}
\hline \multirow[b]{2}{*}{ Subclinical atherosclerosis } & \multicolumn{3}{|c|}{ OR (95\% Cls) } \\
\hline & Model 1 & Model 2 & Model 3 \\
\hline \multicolumn{4}{|l|}{ Carotid atherosclerosis } \\
\hline ACR $\geq 30$ & $1.63(1.36$ to 1.95$)$ & $1.28(1.04$ to 1.59$)$ & 1.07 (0.86 to 1.33$)$ \\
\hline$P_{\text {for trend }}$ & $<0.0001$ & $<0.0001$ & 0.0333 \\
\hline$A C R \geq 30$ & 3.27 (2.75 to 3.90$)$ & 2.57 (2.08 to 3.18$)$ & 1.43 (1.12 to 1.82$)$ \\
\hline$P_{\text {for trend }}$ & $<0.0001$ & $<0.0001$ & 0.0013 \\
\hline \multicolumn{4}{|l|}{ Abnormal $\mathrm{ABI}$} \\
\hline $7.82 \leq \mathrm{ACR}<30$ & $1.00(0.82$ to 1.21$)$ & $0.98(0.80$ to 1.20$)$ & $0.99(0.80$ to 1.21$)$ \\
\hline $7.82 \leq \mathrm{ACR}<30$ & $1.56(1.42$ to 1.73$)$ & 1.35 (1.20 to 1.52$)$ & 1.15 (1.02 to 1.30$)$ \\
\hline$A C R \geq 30$ & 2.31 (1.94 to 2.76) & 2.02 (1.64 to 2.50$)$ & 1.34 (1.07 to 1.68$)$ \\
\hline$P_{\text {for trend }}$ & $<0.0001$ & $<0.0001$ & 0.0017 \\
\hline
\end{tabular}

Subclinical atherosclerosis: (1) carotid atherosclerosis (carotid intima-media thickness $>0.6 \mathrm{~mm}$ or the presence of carotid stenosis as $50 \%$ ), (2) elevated Ba-PWV (Ba-PWV $>1789 \mathrm{~cm} / \mathrm{s})$ or (3) abnormal $\mathrm{ABI}(\mathrm{ABI}<0.9$ or $\mathrm{ABI}>1.4)$.

Model 1: not adjusted; Model 2: adjusted age, sex, body mass index, education level, current smoking, current drinking, physical activity; Model 3: further adjusted triglyceride, high-density lipoprotein cholesterol, low-density lipoprotein cholesterol, total cholesterol, systolic blood pressure, diabetes status, eGFR.

$\mathrm{ABI}$, ankle-brachial index; $\mathrm{ACR}$, albumin-to-creatinine ratio; Ba-PWV, brachial-ankle pulse wave velocity; eGFR, estimated glomerular filtration rate.

adjustment of age, sex, BMI, education level, current smoking, current drinking and physical activity, but the association was attenuated after further adjustments of metabolic risk factors (OR=1.15; 95\% CI 0.999 to 1.33$)$. No correlation was found between low-grade albuminuria and abnormal ABI. When comparing the albuminuria group with the normal group, associations between ACR levels and elevated Ba-PWV (OR=1.43; 95\% CI 1.12 to 1.82), abnormal $\mathrm{ABI}(\mathrm{OR}=1.41 ; 95 \%$ CI 1.03 to 1.94$)$ and presence of subclinical atherosclerosis $(\mathrm{OR}=1.34 ; 95 \%$ CI 1.07 to 1.68 ) were statistically significant in fully adjusted model. As ACR groups grew higher, ORs (95\% CI) for carotid atherosclerosis, elevated Ba-PWV and presence of subclinical atherosclerosis showed rising trends $\left(\mathrm{p}_{\text {for trend }}\right.$ $<0.05)$.

Cox regression analysis showed that higher ACR levels associated with higher risks of incident cardiovascular events and all-cause deaths (table 4). The associations

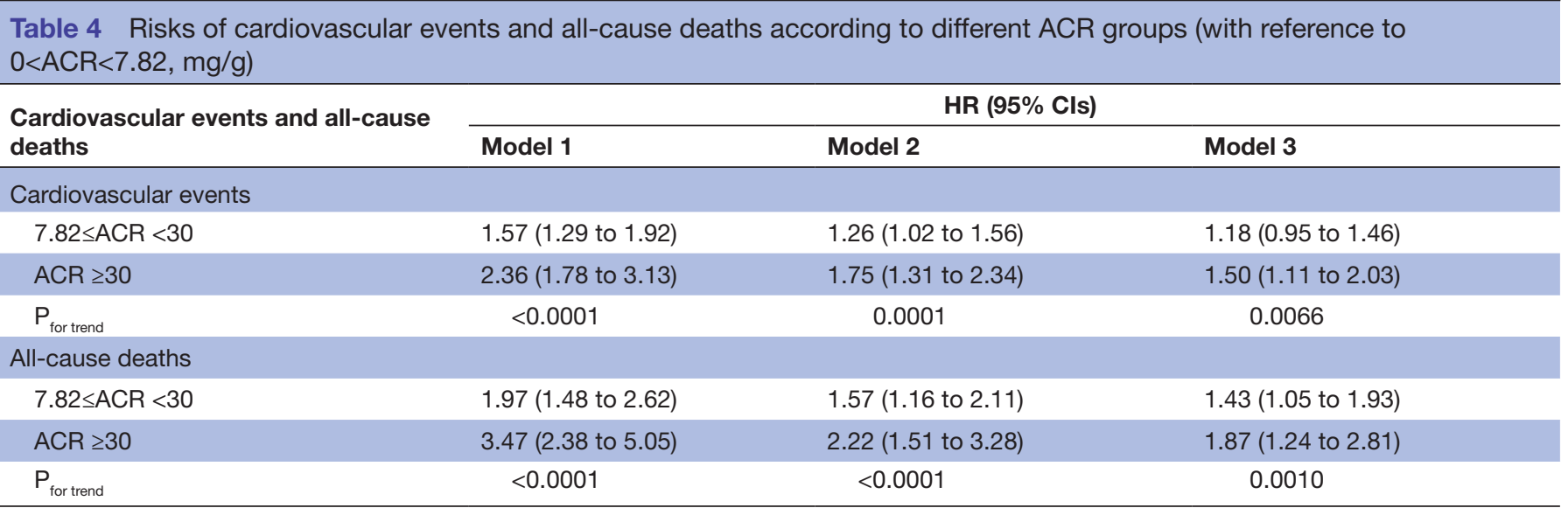

Cardiovascular events: the first instance of nonfatal myocardial infarction, nonfatal stroke, congestive heart failure or cardiovascular-related deaths. Model 1: not adjusted; Model 2: adjusted age, sex, body mass index, education level, current smoking, current drinking, physical activity; Model 3: further adjusted triglyceride, high-density lipoprotein cholesterol, low-density lipoprotein cholesterol, total cholesterol, systolic blood pressure, diabetes status, eGFR.

ACR, albumin-to-creatinine ratio; eGFR, estimated glomerular filtration rate. 
were significant and showed rising tends from the normal group across to the albuminuria group $\left(\mathrm{p}_{\text {for trend }}<0.05\right)$. Comparing to subjects in the normal group, people with low-grade albuminuria had $26 \%$ higher risk $(\mathrm{HR}=1.26$; $95 \%$ CI 1.02 to 1.56 ) of cardiovascular events after adjustment of age, sex, BMI, education level, current smoking, current drinking and physical activity, but the association was attenuated after further adjustments of lipids, blood pressure, diabetes status and eGFR (HR $=1.18 ; 95 \% \mathrm{CI}$ 0.95 to 1.46$)$. People with albuminuria had $50 \%$ higher risk $(\mathrm{HR}=1.50 ; 95 \% \mathrm{CI} 1.11$ to 2.03$)$ of cardiovascular events in multivariate model. For all-cause deaths, people with low-grade albuminuria and albuminuria showed $43 \%$ higher risk $(\mathrm{HR}=1.43 ; 95 \% \mathrm{CI} 1.05$ to 1.93$)$ and $87 \%$ higher risk (HR=1.87; 95\% CI 1.24 to 2.81 ), respectively.

Associations of ACR levels with incident cardiovascular events and all-cause deaths were evaluated according to the subclinical atherosclerosis status (figure 2). In the stratified analysis according to the presence of abnormal ABI, people with albuminuria showed a higher risk of all-cause deaths regardless of ABI status (1.69 (1.06 to 2.70) among category without abnormal ABI; 3.96 (1.32 to 11.89) among category with abnormal ABI), whereas only in those without abnormal ABI, a significant higher risk of cardiovascular events was detected for albuminuria (HR=1.49; 95\% CI 1.08 to 2.07). Besides, the higher risks of cardiovascular events and all-cause deaths with ACR were mainly detected among individuals who had concomitant subclinical atherosclerosis. Compared with subjects in the normal group, the adjusted HRs (95\% CIs) of cardiovascular events for those with albuminuria were 1.63 (1.06 to 2.50) in the category with carotid atherosclerosis, 1.64 (1.11 to 2.41) in the category with elevated Ba-PWV and 1.72 (1.21 to 2.43) in the category with subclinical atherosclerosis, respectively. For all-cause deaths, results in those with subclinical atherosclerosis were similar, but even lowgrade albuminuria showed a significant higher possibility of death. However, these associations were not found in the categories without subclinical atherosclerosis.

Additionally, associations of ACR levels with incident cardiovascular events and all-cause deaths were evaluated according to the diabetes status and number of cardiovascular risk factors (figure 3). Among subjects with diabetes, people with albuminuria had higher risks of cardiovascular events (HR=1.79; 95\% CI 1.12 to 2.84) and all-cause deaths (HR=2.91; 95\% CI 1.49 to 5.66), respectively. The risks were not significant among those without diabetes. When stratified by the number of cardiovascular risk factors, compared with subjects in the normal group, the adjusted HRs (95\% CIs) of cardiovascular events for those with albuminuria were $1.91(0.81$ to 4.50$)$ in the category with $0-2$ cardiovascular risk factors, 1.34 ( 0.71 to $2.53)$ in the category with three cardiovascular risk factors and 1.88 (1.31 to 2.70 ) in the category with 4-6 cardiovascular risk factors, respectively. For all-cause deaths, results in those with 4-6 cardiovascular risk factors were similar (HR=2.85; 95\% CI 1.69 to 4.81 ), but even lowgrade albuminuria showed a significant higher possibility of death ( $\mathrm{HR}=1.67 ; 95 \%$ CI 1.06 to 2.64$)$. However, these associations were not found in the categories with 0-2 cardiovascular risk factors or 3 cardiovascular risk factors, respectively.

\section{DISCUSSION}

The study showed that urinary ACR levels were significantly associated with subclinical atherosclerosis and predicted higher risks of cardiovascular events and allcause deaths independently of glycaemic status and renal function during 4.53 year's follow-up. Of note, the association of higher ACR with risks of cardiovascular events and all-cause deaths was stronger among individuals who had concomitant subclinical atherosclerosis, suggesting the joint impact of the microvascular and macrovascular dysfunctions on the risks of long-term atherosclerotic CVD and death. Additionally, the associations were more significant among subjects with the presence of diabetes or more cardiovascular risk factors.

Although several studies have reported a positive association between ACR and the indicators of subclinical atherosclerosis, most of the investigations have been limited by small sample sizes and incomplete evaluation for subclinical atherosclerosis status. Using data from a well-defined community-based population cohort, our study employed 3 indexes (carotid atherosclerosis, elevated Ba-PWV and peripheral arterial disease (PAD) evaluated by abnormal $\mathrm{ABI}$ ) to reflect the subclinical atherosclerosis status. Our results are consistent with most, but not all, studies that have demonstrated robust associations of ACR with indicators of subclinical atherosclerosis. ${ }^{13-16}$ The inconsistencies might be due to the younger age of our population and lower proportion of underlying metabolic diseases. We found significant positive associations of albuminuria (ACR $\geq 30 \mathrm{mg} / \mathrm{g}$ ) with elevated Ba-PWV and abnormal ABI, whereas a borderline significant relationship was detected for carotid atherosclerosis. Particularly, even normal-high ACR level (low-grade albuminuria) was found to be associated with carotid atherosclerosis after adjustments for other cardiometabolic risk factors. As for elevated Ba-PWV, lowgrade albuminuria showed significantly higher ORs after accounting for sociodemographic characteristics and lifestyle factors. However, the association was weakened after further adjustments of metabolic risk factors, suggesting the mediated effect of metabolic factors. In addition, no significant relationship was detected between low-grade albuminuria and abnormal ABI, which was in line with a study conducted in diabetic patients in China showing the low-grade albuminuria was related to elevated Ba-PWV, but no differences of ABI were detected. ${ }^{8}$

Clinically, carotid atherosclerosis, both elevated CIMT and the presence of carotid plaque, was recognised as a reliable marker of atherosclerosis. Meanwhile, Ba-PWV and $\mathrm{ABI}$ are often used to evaluate the degree of arterial stiffness and to diagnose PAD. American college of cardiology (ACC) and AHA) suggest that early detection 


\section{A CVD events}

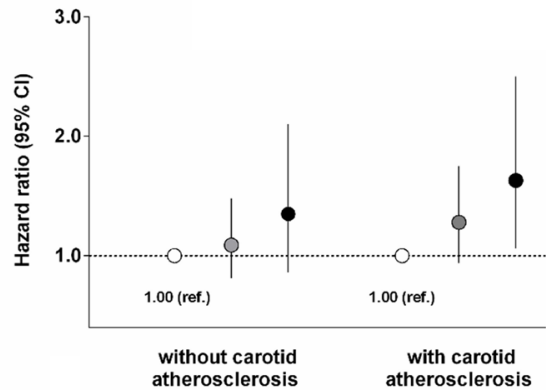

C CVD events

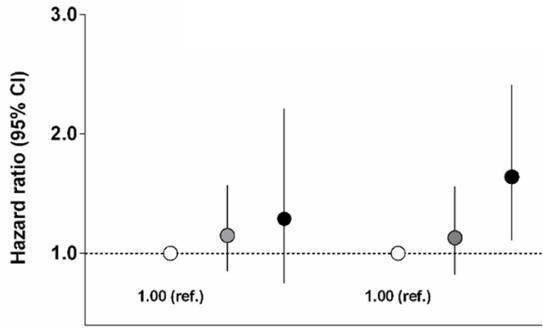

without elevated Ba-PWV with elevated Ba-PWV

\section{E CVD events}

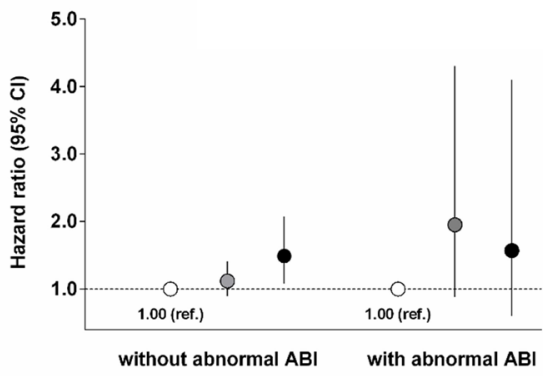

G CVD events

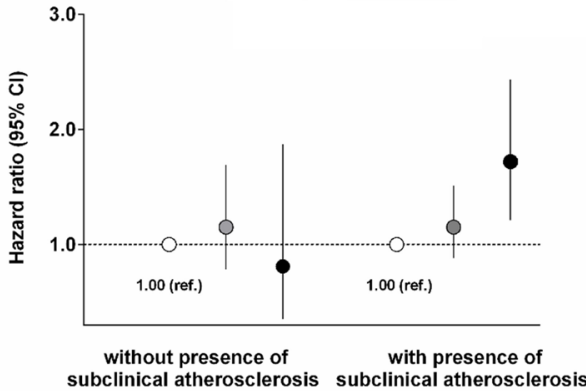

ACR groups
B All-cause deaths

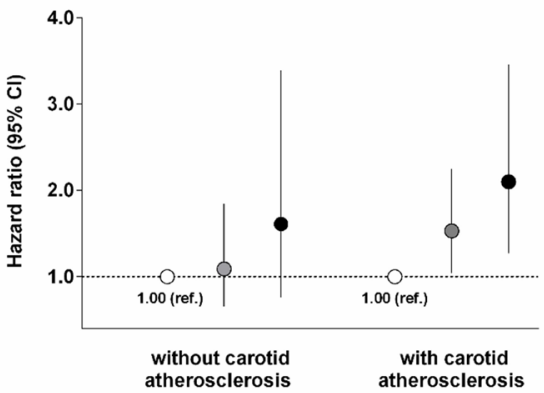

D All-cause deaths

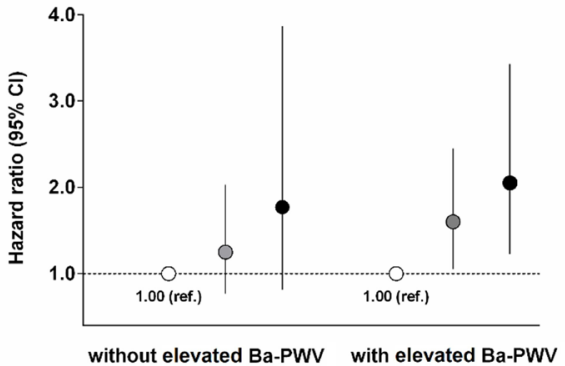

F All-cause deaths

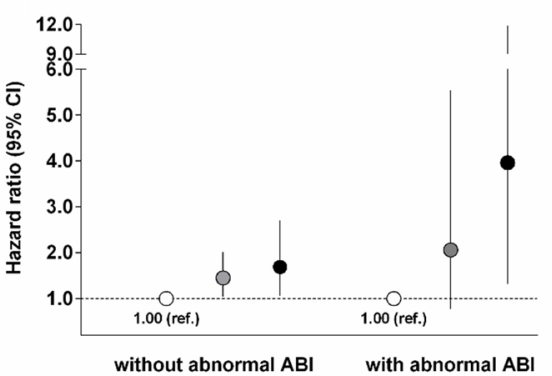

H All-cause deaths

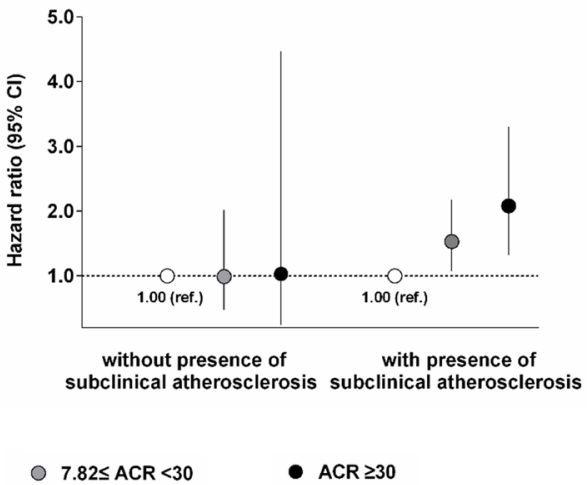

Figure 2 (A-H). Risks of cardiovascular events and all-cause deaths according to different ACR groups stratified by baseline subclinical atherosclerosis. Carotid atherosclerosis (A, B), elevated Ba-PWV (C, D), abnormal ABI (E, F), presence of subclinical atherosclerosis $(G, H)$. The model was adjusted for age, sex, body mass index, education level, current smoking, current drinking, physical activity, triglyceride, high-density lipoprotein cholesterol, low-density lipoprotein cholesterol, total cholesterol, systolic blood pressure, diabetes status, eGFR. ABI, ankle-brachial index; ACR, albumin-to-creatinine ratio; Ba-PWV, brachialankle pulse wave velocity; eFGR, estimated glomerular filtration rate; ref., reference. 
A Cardiovascular events

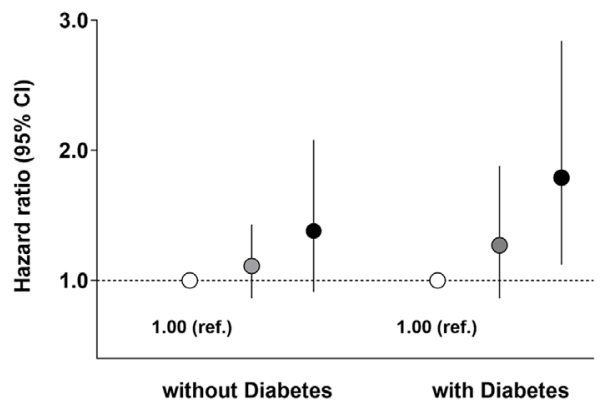

without Diabetes

with Diabetes

C Cardiovascular events
$B^{\text {All-cause deaths }}$

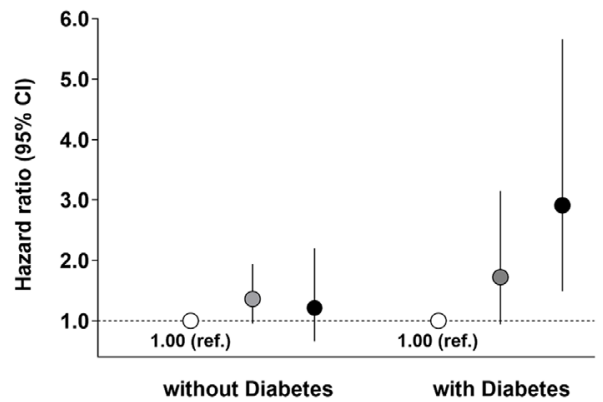

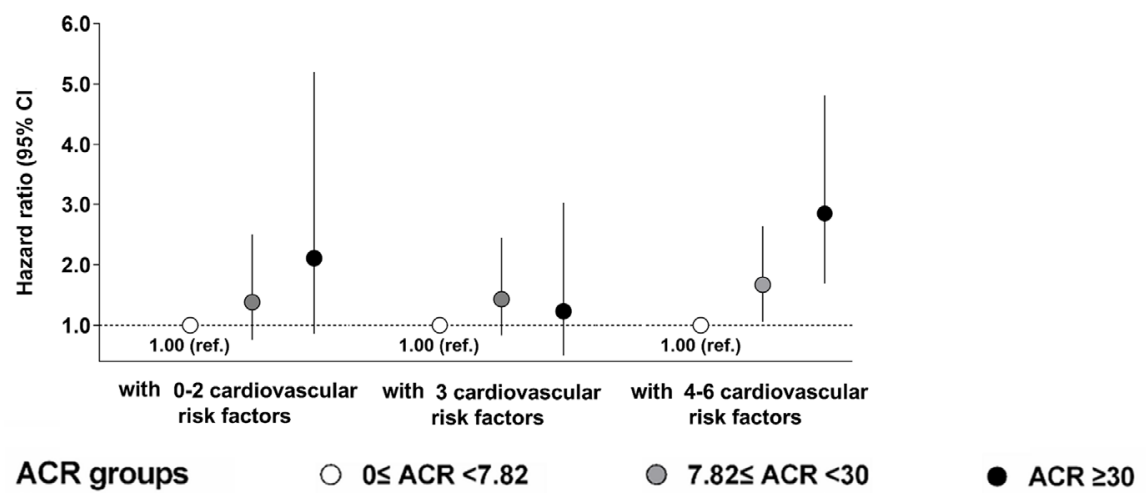

Figure 3 (A-D). Risks of cardiovascular events and all-cause deaths according to different ACR groups stratified by diabetes status and number of cardiovascular risk factors. Diabetes status (A, B), number of cardiovascular risk factors (C, D). The model was adjusted for age, sex, education level, current drinking, eGFR. ACR, albumin-to-creatinine ratio; eGFR, estimated glomerular filtration rate; ref., reference.

and treatment of PAD can prevent disability and reduce mortality. ${ }^{17}$ Subclinical atherosclerosis status can reflect vascular injuries and is regarded as an early stage of atherosclerotic CVD. In our study, we adopted a composite definition of subclinical atherosclerosis using those three indicators and found consistent associations of subclinical atherosclerosis with both low-grade albuminuria and albuminuria, suggesting that ACR levels should be taken into account for vascular disease risk evaluation and prevention. The general standpoint suggested that endothelial dysfunction and subtle chronic inflammation would be the common underlying mechanisms. Other research discovered that accelerated atherosclerosis could be a plausible mechanism for those relationships. ${ }^{18}$

It has been proved that albuminuria is related to the risks of cardiovascular events and all-cause deaths. ${ }^{19} 20$ Our results were consistent with the previous studies. For instance, Ohsawa et al found that albuminuria was useful for predicting cardiovascular events and all-cause deaths in Japanese subjects. ${ }^{21}$ In another study, albuminuria was found independently associated with cardiovascular events and all-cause deaths. ${ }^{22}$ Hence, individuals with albuminuria need to take serious precaution and prevention of cardiovascular events and all-cause deaths. In 
addition, our study showed that low-grade albuminuria increased the risks of cardiovascular events and all-cause deaths, consistent with the previous studies. ${ }^{23}$ As for lowgrade albuminuria, association with cardiovascular events was weakened after further adjustments of metabolic risk factors, suggesting the mediated effect of metabolic factors. Nevertheless, low-grade albuminuria was found to be associated with all-cause deaths significantly. A possible explanation is the potential contribution of low-grade albuminuria to the renal disease-related death. However, the association between low-grade albuminuria and allcause deaths was independent of eGFR level.

To our knowledge, evidence for cardiovascular events risks associated with ACR levels by different subclinical atherosclerosis indicators was still limited. According to our results of baseline analyses between ACR and subclinical atherosclerosis, microvascular and macrovascular impairments usually co-occur and ultimately affect CVD. Thus, we extended the previous knowledge by including the stratified analysis according to subclinical atherosclerosis status and found a stronger association among individuals who had concomitant subclinical atherosclerosis, highlighting the importance of ACR in cardiovascular event risk evaluation, especially in those with pre-existing atherosclerotic abnormalities. Moreover, we found a significantly larger magnitude of the association for albuminuria with cardiovascular events and all-cause deaths in participants with diabetes or more cardiovascular risk factors, which highlight the importance of monitoring and lowering albuminuria for the prevention of CVD events and related deaths in the public health field, especially among subjects with cardiometabolic abnormalities.

A main strength of this study was that it provided a comprehensive evaluation of microvascular and macrovascular indicators and detected the joint impact on the risks of cardiovascular events and all-cause deaths in general population. However, several limitations were identified. First, cross-sectional analysis was applied to investigate the associations between ACR levels and subclinical atherosclerosis, which could not show direct causal links. Second, the follow-up duration was comparatively short and only one follow-up visit was conducted. Third, a relatively small number of participants with cardiovascular events and all-cause deaths were included, so composite outcomes were adopted. Fourth, urinary ACR levels were calculated using a single morning urine sample, instead of 24-hour urine sample. However, the sensitivity and specificity of ACR in predicting microalbuminuria are $87.6 \%$ and $87.5 \%$, respectively. ${ }^{24}$ The use of spot urine collection was recommended and widely applied in population surveys. ${ }^{25}$

In summary, higher urinary ACR levels were positively associated with subclinical atherosclerosis and further predicted the risks of cardiovascular events and all-cause deaths. Evaluation of ACR level should be a high priority for both risk stratification and prevention of cardiovascular events and all-cause deaths, especially among those with pre-existing subclinical atherosclerosis and cardiometabolic abnormalities.

\section{Author affiliations}

${ }^{1}$ Department of Endocrine and Metabolic Diseases, Shanghai Institute of Endocrine and Metabolic Diseases, Ruijin Hospital, Shanghai Jiaotong University School of Medicine, Shanghai, China

${ }^{2}$ Shanghai National Clinical Research Center for Metabolic Diseases, Key Laboratory for Endocrine and Metabolic Diseases of the National Health Commission of the PR China, Shanghai National Center for Translational Medicine, Ruijin Hospital, Shanghai Jiaotong University School of Medicine, Shanghai, China

${ }^{3}$ Clinical Trials Center, Ruijin Hospital, Shanghai Jiaotong University School of Medicine, Shanghai, China

Acknowledgements The authors thank the people in Shanghai, Jiading community for participation.

Contributors SL, JN, SW and ML drafted the manuscript. WW, GN, YB, YX and $M L$ contributed to the planning of the work. $Y B, Y X$ and $M L$ contributed to the conception or design of the work. SL, JN, SW, ZX, ZZ, MX, JL, TW, YC, SW, HL, LY and $M D$ contributed to the conduct and reporting of the work. SL, JN, SW, ZX, ZZ, MX, JL, TW, YC, SW, HL, YX, LY, MD, WW, GN, YB, YX and ML contributed to the acquisition, analysis, or interpretation of data for the work. WW, GN, YB, YX and $M L$ critically revised the manuscript. All gave final approval and agree to be accountable for all aspects of work ensuring integrity and accuracy.

Funding This work was supported by the grants from the National Key R\&D Program of China [2017YFC1310700, 2016YFC1305600, 2016YFC0901200, 2016YFC1305202, 2016YFC1304904, 2018YFC1311800], the National Natural Science Foundation of China [81870560, 81561128019, 81621061, 81700764], National Science and Technology Major Project for 'Significant New Drugs Development' (2017ZX09304007), the Shanghai Municipal Government (18411951800), the Shanghai Shenkang Hospital Development Centre (SHDC12019101), the Shanghai Jiaotong University School of Medicine (DLY201801, 20161301, 20161307), and the Ruijin Hospital (2018CR002). YB was supported by the 'Shanghai Outstanding Academic Leader Programme' and YX was supported by the 'Outstanding Young Talent Programme' from Shanghai Municipal Government.

Competing interests None declared.

Patient and public involvement Patients and/or the public were not involved in the design, or conduct, or reporting, or dissemination plans of this research.

Patient consent for publication Not required.

Provenance and peer review Not commissioned; externally peer reviewed.

Data availability statement Data are available upon reasonable request. Data are available upon reasonable request.

Open access This is an open access article distributed in accordance with the Creative Commons Attribution Non Commercial (CC BY-NC 4.0) license, which permits others to distribute, remix, adapt, build upon this work non-commercially, and license their derivative works on different terms, provided the original work is properly cited, appropriate credit is given, any changes made indicated, and the use is non-commercial. See: http://creativecommons.org/licenses/by-nc/4.0/.

\section{ORCID iD}

Mian Li http://orcid.org/0000-0001-6514-2729

\section{REFERENCES}

1 Gerstein HC, Mann JF, Yi Q, et al. Albuminuria and risk of cardiovascular events, death, and heart failure in diabetic and nondiabetic individuals. JAMA 2001;286:421-6.

2 Yokoyama H, Araki S, Haneda M, et al. Chronic kidney disease categories and renal-cardiovascular outcomes in type 2 diabetes without prevalent cardiovascular disease: a prospective cohort study (JDDM25). Diabetologia 2012;55:1911-8.

3 Arnlöv J, Evans JC, Meigs JB, et al. Low-grade albuminuria and incidence of cardiovascular disease events in nonhypertensive and nondiabetic individuals: the Framingham heart study. Circulation 2005;112:969-75.

4 SS H, Gao RL, Liu YS. Profile of China's cardiovascular disease report 2018. Chin Circ J 2019;34:209-20. 
5 Sarnak MJ, Levey AS, Schoolwerth AC, et al. Kidney disease as a risk factor for development of cardiovascular disease: a statement from the American heart association councils on kidney in cardiovascular disease, high blood pressure research, clinical cardiology, and epidemiology and prevention. Circulation 2003;108:2154-69.

$6 \mathrm{Ma} \mathrm{H}$, Lin $\mathrm{H}$, Hofman A, et al. Low-grade albuminuria is associated with carotid atherosclerosis in normotensive and euglycemic Chinese middle-aged and elderly adults: the Shanghai Changfeng study. Atherosclerosis 2013;228:237-42.

7 Yoon HE, Kim ES, Mo EY, et al. High normal albuminuria is associated with arterial stiffness and carotid atherosclerosis in Korean patients with type 2 diabetes. Nutr Metab Cardiovasc Dis 2015;25:787-94.

8 Huang L, Yang L, Zhang S, et al. Low-grade albuminuria associated with brachial-ankle pulse wave velocity in young adults with type 2 diabetes mellitus in China. Diabetes Metab Res Rev 2015;31:262-8.

9 Li M, Xu Y, Xu M, et al. Association between nonalcoholic fatty liver disease (NAFLD) and osteoporotic fracture in middle-aged and elderly Chinese. J Clin Endocrinol Metab 2012;97:2033-8.

10 Kang SH, Cho KH, Do JY. Non-alcoholic fatty liver disease is associated with low-grade albuminuria in men without diabetes mellitus. Int J Med Sci 2019;16:285-91.

11 Craig CL, Marshall AL, Sjöström M, et al. International physical activity questionnaire: 12-country reliability and validity. Med Sci Sports Exerc 2003;35:1381-95.

12 Lloyd-Jones DM, Hong Y, Labarthe D, et al. Defining and setting national goals for cardiovascular health promotion and disease reduction: the American heart association's strategic impact goal through 2020 and beyond. Circulation 2010;121:586-613.

13 Kweon S-S, Shin M-H, Lee Y-H, et al. Higher normal ranges of urine albumin-to-creatinine ratio are independently associated with carotid intima-media thickness. Cardiovasc Diabetol 2012;11:112.

14 Kong X, Jia X, Wei Y, et al. Association between microalbuminuria and subclinical atherosclerosis evaluated by carotid artery intimamedia in elderly patients with normal renal function. BMC Nephrol 2012;13:37.

$15 \mathrm{Xu} \mathrm{X}, \mathrm{He} \mathrm{J}$, Wang S, et al. Ankle-brachial index and brachial-ankle pulse wave velocity are associated with albuminuria in communitybased Han Chinese. Clin Exp Hypertens 2016;38:618-23.
$16 \mathrm{Xu} \mathrm{B}$, Dai M, Li M, et al. Low-grade albuminuria is associated with peripheral artery disease in Chinese diabetic patients. Atherosclerosis 2014;232:285-8.

17 Hirsch AT, Haskal ZJ, Hertzer NR, et al. ACC/AHA 2005 practice guidelines for the management of patients with peripheral arterial disease (lower extremity, renal, mesenteric, and abdominal aortic). Circulation 2006;113:e463-654.

18 Stehouwer CDA, Smulders YM. Microalbuminuria and risk for cardiovascular disease: analysis of potential mechanisms. J Am Soc Nephrol 2006;17:2106-11.

19 Matsushita K, Coresh J, Sang Y, et al. Estimated glomerular filtration rate and albuminuria for prediction of cardiovascular outcomes: a collaborative meta-analysis of individual participant data. Lancet Diabetes Endocrinol 2015;3:514-25.

20 Chronic Kidney Disease Prognosis Consortium, Matsushita K, van der Velde $\mathrm{M}$, et al. Association of estimated glomerular filtration rate and albuminuria with all-cause and cardiovascular mortality in general population cohorts: a collaborative meta-analysis. Lancet 2010;375:2073-81.

21 Ohsawa M, Fujioka T, Ogasawara K, et al. High risks of all-cause and cardiovascular deaths in apparently healthy middle-aged people with preserved glomerular filtration rate and albuminuria: a prospective cohort study. Int J Cardiol 2013;170:167-72.

22 Weiner DE, Park M, Tighiouart $\mathrm{H}$, et al. Albuminuria and allograft failure, cardiovascular disease events, and all-cause death in stable kidney transplant recipients: a cohort analysis of the FAVORIT trial. Am J Kidney Dis 2019;73:51-61.

23 Tanaka F, Komi R, Makita S, et al. Low-grade albuminuria and incidence of cardiovascular disease and all-cause mortality in nondiabetic and normotensive individuals. J Hypertens 2016;34:506-12.

24 Witte EC, Lambers Heerspink HJ, de Zeeuw D, et al. First morning voids are more reliable than spot urine samples to assess microalbuminuria. J Am Soc Nephrol 2009;20:436-43.

25 Gansevoort RT, Verhave JC, Hillege HL, et al. The validity of screening based on spot morning urine samples to detect subjects with microalbuminuria in the general population. Kidney Int Supp/ 2005;67:S28-35. 